\title{
Bernard Soulier Syndrome: Presenting As High-grade Fever In A Young Male
}

\author{
Syed Parvez Asghar, Rida Ali, Syed Sajid Abbas Jaffari, Zehra Akhtar, Naveed Siddique
}

\begin{abstract}
:
Bernard-Soulier syndrome (BSS) is a rare inherited disorder of blood clotting (coagulation). They may be life-threatening and demand immediate attention. Congenital bleeding disorders especially pose a diagnostic challenge to the clinician because of their rarity and the need to be differentiated from the other common causes of bleeding in children. We present a case of young male presenting with generalized weakness, high grade fever with chills, dry cough and black tarry stools (2-3 stools/day). No response to steroids and further evaluation by platelet, ADP, collegen, epinephrine and defective response to ristocetin led to the correct diagnosis - Bernard soulier syndrome (BSS). However, it is imperative to have arrived at correct diagnosis in order to save unnecessary therapy and to take due precautions for prevention of bleeding.
\end{abstract}

Key Words: Bernard Soulier Syndrome, Bleeding Disorder, Decrease expression of glycoprotein.

\section{INTRODUCTION:}

It is congenital bleeding disorder, named after two physicians Dr. Jean Pierre Soulier in 1948. It was recognized to be familial and inherited in autosomal recessive manner. It is characterized by prolonged bleeding time, a low platelet count and giant platelet membrane glycoprotein GPIb. Patient usually present in neonate, infancy, early childhood with bruises, nose bleed (epistaxis) or gingival bleeding. Later in adolescence it may present as menorrhagia in females, stomach ulcer, trauma and surgery.

\section{CASE REPORT:}

An 18 year young male student of class X, presented on 19 DEC, 2009 with generalized weakness, high grade fever with chills, dry cough and black tarry stools (2-3 stools/day).His past medical history revealed prolonged bleeding after circumcision, sports injury, epistaxis and malena .He had various hospital admissions and blood transfusion history. Bleeding usually provoked by aspirin and panadol intake.

Upon clinical examination, he was pale and rest of examination is unremarkable. The patient was thoroughly investigated by blood tests, radiological studies, flow

Syed Parvez Asghar,

Professor, Medicine Department

I Bahria University Medical and Dental College, Karachi

I Rida Ali,

I Registrar, Medicine Department

I Bahria University Medical and Dental College, Karachi

I Syed Sajid Abbas Jaffari,

I Professor, Head of Medicine Department

Bahria University Medical and Dental College, Karachi

Zehra Akhtar,

Lecturer, Medicine Department

I Bahria University Medical and Dental College, Karachi

Naveed Siddique

I Lecturer, Medicine Department

I Bahria University Medical and Dental College, Karachi

I Received: 22-01-2019

Accepted: 15-05-2019

1 cytometry, and platelet aggregation studies. His BCP shows $\mathrm{Hb}: 6.9 \mathrm{~g} / \mathrm{dl}$, TLC:12.8×/L,LFTs: bilirubin:18 $\mu / \mathrm{L}, \mathrm{ALT}: 49 \mu / \mathrm{L}$, ALK.PHOSPHATASE: $108 \mu / \mathrm{L}$, PT/APTT are normal. Bleeding time : more than $7.00 \mathrm{~min} / \mathrm{sec}$, retic count: $05 \%$ to $11 \%$, combs test direct/indirect: -ve,HbsAg: -ve ,Anti HCV : -ve, ferritin:83.5ng/ml, ANA: -ve, D-DIMER: normal , stool for occult blood : +ve, DENGUE VIRUS IgM: -ve. Peripheral blood film shows: Dimorphic picture, anisocytosis, poikilocytosis. Polychromasia, few lymphoctes, microcytes, target cells,right shift neutrophills, spherocytes, large platelet seen.

Oesogastoduodenoscopy shows multiple bleeding points at $1^{\text {st }}$ and $2^{\text {nd }}$ part of duodenum with multiple polyposis, which was found to be benign polyp. Ultrasound and CT-SCAN abdomen is normal.

Platelet aggregation studies shows: normal response to ADP, Collagen, Epinephrine and Defective response to Ristocetin. Von-willebrand AG-factor $=119 \%$. Risticetin co-factor $=115 \%$

All above findings are suggestive of BERBARD SOULIER SNDROME.(BSS)

This patient was treated symptomatically with proton pump inhibitors, folic acids, iron supplements, antibiotics, electrolyte replacement and blood transfusion. Injection NOVASEVEN $(1.2 \mathrm{mg}$ ) vials $2.4 \mathrm{mg}$ IV at interval of $3 \mathrm{hrs,}$ started after platelet aggregation study which did not responded by our patient.

Desmopressin nasal spray was started twice a day and results were poitive. His BCP shows $\mathrm{Hb}: 11.2 \mathrm{~g} / \mathrm{dl}$, TLC:10.6×10^9/L, LFTs: serum biirubin:35, ALT:29, serum ALK.PHS:189 $\mu /$, PT:16, APTT:34, stool for occult blood : -ve. Follow up at 6 monthly was advised that reveals good results.

\section{DISCUSSION:}

Bernard-Soulier syndrome is a bleeding disorder associated with abnormal platelets, which are blood cell fragments involved in blood clotting. In affected individuals, platelets are unusually large and fewer in number than usual (a combination known as macrothrombo-cytopenia). People 
Bernard Soulier Syndrome: Presenting As High-Grade Fever In A Young Male

with Bernard-Soulier syndrome tend to bruise easily and have an increased risk of nosebleeds (epistaxis). They may

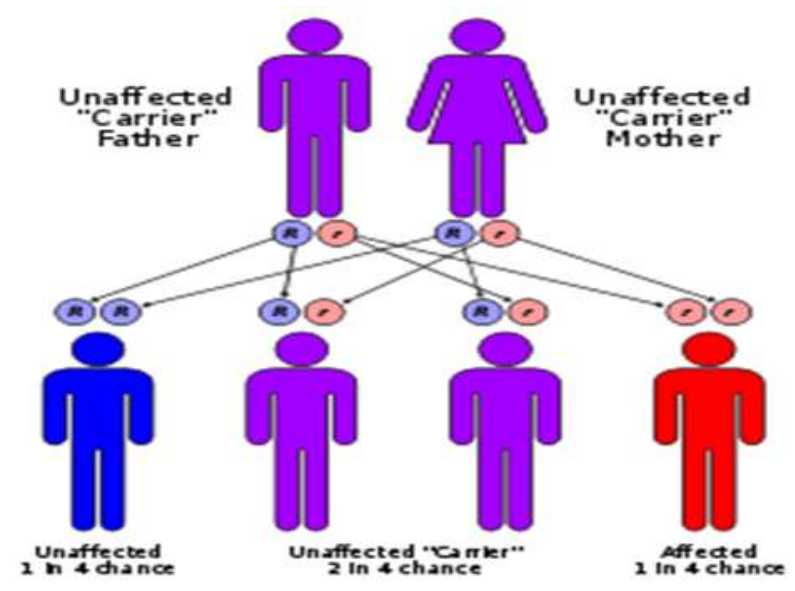

also experience abnormally heavy or prolonged bleeding following minor injury or surgery or even without trauma (spontaneous bleeding). In some affected individuals, bleeding under the skin causes tiny red or purple spots on the skin called petechiae. Women with Bernard-Soulier syndrome often have heavy or prolonged menstrual periods (menorrhagia).

Bernard soulier syndrome (BSS) is a qualitative and quantitative bleeding disorder that is characterized by the absence or decrease expression of glycoprotien GPIX/V complexs on the surface of platelet that is the binding receptor of vWF for platelet at the site of vascular injury. This absence of platelet plug formation results in increase bleeding tendency. It has three genetic variants which are: $\mathrm{i}$ : Type AGP1BA, ii: Type B-GP1BB and iii: type C-GP9. BernardSoulier syndrome has an autosomal recessive pattern of inheritance.

The GPIb-IX-V complexes are four proteins GPIb alpha, GPIb beta and GPV, present on the surface of platelet. The subunit GPIb alpha binds to a protein vWF to initiate clotting mechanism. If vWF protein also deficient, it would result in other bleeding disorder such as hemophilia A or B. The other conditions of bleeding disorders are as follows.

The disease is found in whites of European ancestry, Japanese other ethnic group is uncommon, with occurrence rate less than $1 \mathrm{case} / \mathrm{million}$ population among both gender. Patient with BSS, gives history of prolonged bleeding after any tooth extraction, trauma, surgery, bleeding after circumcision in neonate, bruise and mucosal bleeding on physical examination. Family history of any bleeding disorder should be asked in detail by the patient, as homozygote presents with prolonged bleeding episode, while heterozygote do not usually manifest bleeding.

Laboratory investigation include CBC (thrombocytopenia), peripheral blood smear shows gaint platelet that is often mistaken for RBCs on automatic counters as platelet exceeds the size of RBCs.Bleeding time is prolonged analyzed by automatic platelet function analyzer (PFA)PFA-100.Platelet aggregation studies show normal response to ADP, epinephrine, collagen but do not aggregate in response to Ristocetin, this is not corrected by the addition of normal plasma that differentiate it from vWD. Flow cytometry shows abnormalities of platelet membrane glycoprotein.

There is no cure for BSS, although supportive and medical care is available. Educate the patient about the disease and need to avoid trauma, in contact with sports, surgery, emphasize on good oral hygiene. Avoid anti-platelet drugs such as aspirin, NSAIDs, ibruphen, naproxen, iron supplements can be started in women with menorrahgia as

\begin{tabular}{|l|l|c|c|c|}
\hline \multicolumn{1}{|c|}{ Conditions } & $\begin{array}{c}\text { Prothrombin } \\
\text { Time }\end{array}$ & $\begin{array}{c}\text { Partial thromb- } \\
\text { oplastin time }\end{array}$ & $\begin{array}{c}\text { Bleeding } \\
\text { Time }\end{array}$ & $\begin{array}{c}\text { Platelet } \\
\text { Count }\end{array}$ \\
\hline Vitamin K deficiency or Warfarin & Prolonged & Prolonged & Unaffected & Unaffected \\
\hline Disseminated intravascular coagulation & Prolonged & Prolonged & Prolonged & Decreased \\
\hline Von Willebrand disease & Unaffected & Prolonged & Prolonged & Unaffected \\
\hline Hemophilia & Unaffected & Prolonged & Unaffected & Unaffected \\
\hline Aspirin & Unaffected & Unaffected & Prolonged & Unaffected \\
\hline Thrombocytopenia & Unaffected & Unaffected & Prolonged & Decreased \\
\hline Early liver failure & Prolonged & Unaffected & Unaffected & Unaffected \\
\hline End Stage Liver failure & Prolonged & Prolonged & Prolonged & Decreased \\
\hline Uremia & Unaffected & Unaffected & Prolonged & Unaffected \\
\hline Congenital a fibrinogenemia & Prolonged & Prolonged & Prolonged & Unaffected \\
\hline Factor V deficiency & Prolonged & Prolonged & Unaffected & Unaffected \\
\hline Factor X deficiency as seen in amyloid purpura & Prolonged & Prolonged & Unaffected & Unaffected \\
\hline Glanzmann's thrombasthenia & Unaffected & Unaffected & Prolonged & Unaffected \\
\hline Bernard Soulier syndrome & Unaffected & Unaffected & Prolonged & Decreased \\
\hline
\end{tabular}


to cover low iron stores that results due to prolonged bleeding tendency in BSS. Medical treatment of bleeding episode includes anti-fibrinolytic agents such as aminocaproic acid, or traxemic acid useful for mucosal bleeding. Platelet transfusion is the only available therapy for surgery or life threatening hemorrhage. Although the patient may develop antiplatelet antibodies due to GPIb/IX/V against transfused platelet.

DDAVP, have been shown to shorten the B.T. in some but not in all patients. It shorten the bleeding episodes by increasing level of vWF binding to some residual GPIb in patients without an absolute deficiency. Its dose is same in adults and pediatric $0.3 \mathrm{mcg} / \mathrm{kg} \mathrm{IV}$, intranasal $<50 \mathrm{~kg}: 150$ $\mathrm{mcg}$, $>50 \mathrm{~kg}: 300 \mathrm{mcg}$ (one spray in each nostril).In our case , patient is also well responded to DDAVP(NASAL SPRAY) rather than recombinant factor VII.

\section{CONCLUSION:}

Recombinant activated factor VII(NOVOSEVEN) is avitamin-k dependent GP indicated for bleeding episodes such as Hemophilia A/B and inhibitors. It works by activating the extrinsic pathway of the coagulation cascade, forming complexes with tissue factor and promoting factor $\mathrm{X}$ to $\mathrm{Xa}$, factor IX to IXa and factor II to IIa. Although it is limited in patients with congenital platelet disorders. As in our patient its use does not manifest good results, besides this DDAVP works well. However researches are studying the efficacy of bone marrow transplantations and have shown some success in treating the patients with BSS.

\section{REFERENCES:}

1. Lanza F (2006). "Bernard-Soulier syndrome (hemorrhagiparous thrombocytic dystrophy)". Orphanet J Rare Dis.16 (1): 46. doi:10.1186/1750-1172-1-46. PMC 1660532. PMID 17109744.

2. Anesthetic and perioperative management of a patient with Bernard-Soulier syndrome Georgia Kostopanagiotou MD, IoannaSiafakaMDa, ConstantinosSikiotisMDa, and Vassilios SmyrniotisMDa. Received 23 July 2003; Revised 21 October 2003

3. Pham A, Wang J. "Bernard-Soulier syndrome: an inherited platelet disorder" (subscription required). Arch. Pathol. Lab. Med. 2007;131(12):1834-6. PMID 18081445.

4. synd/2075 at Who Named It?

5. Bernard J, Soulier JP (December 1948). "[Sur une nouvelle variété de dystrophiethrombocytairehémorragiparecongénitale]" (in French). Semaine des hôpitaux de Paris24 (Spec. No.): 3217-23. PMID 18116504.

6. Online 'Mendelian Inheritance in Man' (OMIM) GIANT PLATELET SYNDROME -231200.

7. Almeida AM,KhairK,Hann I, et al.The use of recombinant factor VIIA in children with inherited platelet function disorders. Br J Haematol2003;121:477-81.

8. Peters M, HeijboerH.Treatment of a patient with Bernard Soulier Syndrome and recurrent nosebleeds with recombinant factor VIIa.ThrombHaemost 1998;80:352.

9. De Marco L, Mazzucato M, Fabris F, et al. (July 1990). "Variant Bernard-Soulier syndrome type bolzano. A congenital bleeding disorder due to a structural and functional abnormality of the platelet glycoprotein Ib-IX complex". J. Clin. Invest.86 (1): 25-31. doi:10.1172/JCI114692. PMC 296685. PMID 1694864

10. Giant platelet syndrome; Bernard-Soulier syndrome; Deficiency of Platelet glycoprotein 1b at NIH's Office of Rare Diseases. 